\title{
Norm of an integral operator on some analytic function spaces on the unit disk
}

Hao $\mathrm{Li}^{1}$ and Songxiao $\mathrm{Li}^{2 *}$

"Correspondence: jyulsx@163.com

2Department of Mathematics,

JiaYing University, Meizhou,

GuangDong 514015, China

Full list of author information is

available at the end of the article

\section{Abstract}

If $f$ is an analytic function in the unit disc $\mathbb{D}$, a class of integral operators is defined as follows:

$$
I_{f}(h)(z)=\int_{0}^{z} f(w) h^{\prime}(w) d w, \quad h \in H(\mathbb{D}), z \in \mathbb{D}
$$

The norm of $I_{f}$ on some analytic function spaces is computed in this paper.

MSC: Primary 47B38; secondary 32A35

Keywords: norm; integral operator; analytic function space

\section{Introduction}

Let $\mathbb{D}=\{z:|z|<1\}$ be the unit disk of a complex plane $\mathbb{C}$. Denote by $H(\mathbb{D})$ the class of functions analytic in $\mathbb{D}$. Let $d \sigma$ denote the normalized Lebesgue area measure in $\mathbb{D}$ and $g(a, z)$ the Green function with logarithmic singularity at $a$, i.e., $g(a, z)=-\log \left|\varphi_{a}(z)\right|$, where $\varphi_{a}(z)=(a-z) /(1-\bar{a} z)$ is the Möbius transformation of $\mathbb{D}$.

For $0<p<\infty$, the $Q_{p}$ is the space of all functions $f \in H(\mathbb{D})$, for which

$$
\|f\|_{Q_{p}}^{2}=|f(0)|^{2}+\sup _{a \in \mathbb{D}} \int_{\mathbb{D}}\left|f^{\prime}(z)\right|^{2}\left(1-\left|\varphi_{a}(z)\right|^{2}\right)^{p} d \sigma(z)<\infty .
$$

We know that $Q_{1}=\mathrm{BMOA}$, the space of all analytic functions of bounded mean oscillation $[1,2]$. For all $p>1$, the space $Q_{p}$ is the same and equal to the Bloch space $\mathfrak{B}$, consisting of analytic functions $f$ in $\mathbb{D}$ such that

$$
\|f\|_{\mathfrak{B}}=|f(0)|+\sup _{z \in \mathbb{D}}\left|f^{\prime}(z)\right|\left(1-|z|^{2}\right)<\infty .
$$

See $[3,4]$ for the theory of Bloch functions.

For $\alpha>0$, the $\alpha$-Bloch space, denoted by $\mathfrak{B}^{\alpha}$, is the space of all functions $f$ in $\mathbb{D}$, for which

$$
\|f\|_{\mathfrak{B}^{\alpha}}=|f(0)|+\sup _{z \in \mathbb{D}}\left|f^{\prime}(z)\right|\left(1-|z|^{2}\right)^{\alpha}<\infty .
$$

Obviously, $\mathfrak{B}^{\alpha_{1}} \varsubsetneqq \mathfrak{B} \varsubsetneqq \mathfrak{B}^{\alpha_{2}}$ for $0<\alpha_{1}<1<\alpha_{2}<\infty$.

\section{Springer}

( $2013 \mathrm{Li}$ and Li; licensee Springer. This is an Open Access article distributed under the terms of the Creative Commons Attribution License (http://creativecommons.org/licenses/by/2.0), which permits unrestricted use, distribution, and reproduction in any medium, provided the original work is properly cited. 
For any $f \in H(\mathbb{D})$, the next two integral operators on $H(\mathbb{D})$ are induced as follows:

$$
I_{f}(h)(z)=\int_{0}^{z} h^{\prime}(w) f(w) d w \quad \text { and } \quad J_{f}(h)(z)=\int_{0}^{z} h(w) f^{\prime}(w) d w \quad(z \in \mathbb{D}) .
$$

Let $M_{f}$ denote the multiplication operator, that is, $M_{f}(h)=f h$.

Let $f \in H(\mathbb{D})$. Then

$$
\left(I_{f}+J_{f}\right) h=f h-f(0) h(0)=M_{f}(h)-f(0) h(0) .
$$

If $f$ is a constant, then all results about $I_{f}, J_{f}$ or $M_{f}$ are trivial. In general, $f$ is assumed to be non-constant. Both integral operators have been studied by many authors. See [5-21] and the references therein.

Norm of composition operator, weighted composition operator and some integral operators have been studied extensively by many authors, see [22-34] for example. Recently, Liu and Xiong discussed the norm of integral operators $I_{f}$ and $J_{f}$ on the Bloch space, Dirichlet space, BMOA space and so on in [35].

In this paper, we study the norm of integral operator $I_{f}$. The norm of $I_{f}$ on several analytic function spaces is computed.

\section{Main results}

In this section, we state and prove our main results. In order to formulate our main results, we need an auxiliary result which is incorporated in the following lemma.

Lemma 2.1 Let $0<p<1$. For any $z_{0} \in \mathbb{D}$, the function

$$
g_{z_{0}}(z)=\frac{z_{0}-z}{1-\bar{z}_{0} z}-z_{0}
$$

is analytic in $\mathbb{D}$ and $\left\|g_{z_{0}}\right\|_{Q_{p}}=1 /(p+1)^{1 / 2}$.

Proof By (1.1) and [1, Proposition 1, p.109], we have

$$
\begin{aligned}
\left\|g_{z_{0}}\right\|_{Q_{p}}^{2} & =\sup _{a \in \mathbb{D}} \int_{\mathbb{D}}\left|g_{z_{0}}^{\prime}(z)\right|^{2}\left(1-\left|\varphi_{a}(z)\right|^{2}\right)^{p} d \sigma(z) \\
& =\sup _{b \in \mathbb{D}} \int_{\mathbb{D}}\left(1-\left|\varphi_{b}(z)\right|^{2}\right)^{p} d \sigma(z),
\end{aligned}
$$

where $b=\varphi_{z_{0}}(a)$. Taking $w=\varphi_{b}(z)$, we have

$$
\left\|g_{z_{0}}\right\|_{Q_{p}}^{2}=\sup _{b \in \mathbb{D}}\left(1-|b|^{2}\right)^{2} \int_{\mathbb{D}} \frac{\left(1-|w|^{2}\right)^{p}}{|1-\bar{b} w|^{4}} d \sigma(w) .
$$

Since

$$
\frac{1}{(1-\bar{b} w)^{2}}=\sum_{n=0}^{\infty} \frac{\Gamma(n+2)}{n ! \Gamma(2)} \bar{b}^{n} w^{n}=\sum_{n=0}^{\infty} \frac{\Gamma(n+2)}{n !} \bar{b}^{n} w^{n}
$$


we have

$$
\begin{aligned}
\int_{\mathbb{D}} \frac{\left(1-|w|^{2}\right)^{p}}{|1-\bar{b} w|^{4}} d \sigma(w) & =\sum_{n=0}^{+\infty} \frac{\Gamma(n+2)^{2}}{(n !)^{2}}|b|^{2 n} \int_{\mathbb{D}}\left(1-|w|^{2}\right)^{p}|w|^{2 n} d \sigma(w) \\
& =\sum_{n=0}^{+\infty} \frac{\Gamma(n+2)^{2}}{(n !)^{2}}|b|^{2 n} \int_{0}^{1}(1-r)^{p} r^{n} d r \\
& =\sum_{n=0}^{+\infty} \frac{\Gamma(n+2)^{2}}{(n !)^{2}} \frac{\Gamma(p+1) \Gamma(n+1)}{\Gamma(n+p+2)}|b|^{2 n} \\
& =\sum_{n=0}^{+\infty} \frac{\Gamma(p+1) \Gamma(n+2)^{2}}{n ! \Gamma(n+p+2)}|b|^{2 n} .
\end{aligned}
$$

A simple computation shows

$$
\frac{\Gamma(p+1) \Gamma(n+2)^{2}}{n ! \Gamma(n+p+2)}=\frac{(n+1) !(n+1)}{(p+1)(p+2) \cdots(p+n+1)} .
$$

Also, it is easy to see

$$
\frac{1}{p+1} \leq \frac{n+1}{p+n+1} \leq \frac{(n+1) !(n+1)}{(p+1)(p+2) \cdots(p+n+1)} \leq \frac{n+1}{p+1} .
$$

Thus,

$$
\left\|g_{z_{0}}\right\|_{Q_{p}}^{2} \leq \sup _{b \in \mathbb{D}} \frac{\left(1-|b|^{2}\right)^{2}}{p+1} \sum_{n=0}^{+\infty}(n+1)|b|^{2 n}=\sup _{b \in \mathbb{D}} \frac{\left(1-|b|^{2}\right)^{2}}{p+1} \frac{1}{\left(1-|b|^{2}\right)^{2}}=\frac{1}{p+1}
$$

and

$$
\left\|g_{z_{0}}\right\|_{Q_{p}}^{2} \geq \sup _{b \in \mathbb{D}} \frac{\left(1-|b|^{2}\right)^{2}}{p+1} \sum_{n=0}^{+\infty}|b|^{2 n}=\sup _{b \in \mathbb{D}} \frac{\left(1-|b|^{2}\right)^{2}}{p+1} \frac{1}{1-|b|^{2}}=\frac{1}{p+1}
$$

Then the proof is complete.

First, we consider the norm of $I_{f}$ on $Q_{p}, 0<p<1$.

Theorem 2.2 Let $0<p<1$. If $f \in H(\mathbb{D})$, then $I_{f}$ is bounded on $Q_{p}$ if and only if $f \in H^{\infty}$. Moreover,

$$
\left\|I_{f}\right\|=\|f\|_{H^{\infty}} .
$$

Proof For any $h \in Q_{p}$ with $\|h\|_{Q_{p}}=1$, it is trivial that $\left\|I_{f}\right\| \leq\|f\|_{H^{\infty}}$. To prove the converse, define $c=\sup _{z \in \mathbb{D}}|f(z)|$. Given any $\epsilon>0$, there exists $z_{1} \in \mathbb{D}$ such that $\left|f\left(z_{1}\right)\right|>c-\epsilon$. Let $h(z)=g_{z_{1}}(z) /\left\|g_{z_{1}}\right\|_{Q_{p}}$, where

$$
g_{z_{1}}(z)=\frac{z_{1}-z}{1-\bar{z}_{1} z}-z_{1} .
$$


It is easy to see that

$$
\|h\|_{Q_{p}}=1, \quad\left|h^{\prime}\left(z_{1}\right)\right|\left(1-\left|z_{1}\right|^{2}\right)=1 /\left\|g_{z_{1}}\right\|_{Q_{p}} .
$$

Henceforth,

$$
\begin{aligned}
\left\|I_{f}\right\|^{2} & \geq\left\|I_{f} h\right\|_{Q_{p}}^{2}=\sup _{a \in \mathbb{D}} \int_{\mathbb{D}}\left|h^{\prime}(z) f(z)\right|^{2}\left(1-\left|\varphi_{a}(z)\right|^{2}\right)^{p} d \sigma(z) \\
& =\sup _{a \in \mathbb{D}} \int_{\mathbb{D}}\left|h^{\prime}\left(\varphi_{a}(w)\right) f\left(\varphi_{a}(w)\right) \varphi_{a}^{\prime}(w)\right|^{2}\left(1-|w|^{2}\right)^{p} d \sigma(w) .
\end{aligned}
$$

Taking $w=r e^{i \theta}$ and by the subharmonicity of $\left|h^{\prime}\left(\varphi_{a}(w)\right) f\left(\varphi_{a}(w)\right) \varphi_{a}^{\prime}(w)\right|^{2}$, we obtain

$$
\begin{aligned}
\left\|I_{f}\right\|^{2} & \geq \sup _{a \in \mathbb{D}} \int_{\mathbb{D}}\left|h^{\prime}(z) f(z)\right|^{2}\left(1-\left|\varphi_{a}(z)\right|^{2}\right)^{p} d \sigma(z) \\
& =\sup _{a \in \mathbb{D}} \int_{0}^{1} \frac{1}{\pi} \int_{0}^{2 \pi}\left|h^{\prime}\left(\varphi_{a}\left(r e^{i \theta}\right)\right) f\left(\varphi_{a}\left(r e^{i \theta}\right)\right) \varphi_{a}^{\prime}\left(r e^{i \theta}\right)\right|^{2}\left(1-r^{2}\right)^{p} r d r d \theta \\
& \geq \sup _{a \in \mathbb{D}}\left|h^{\prime}(a) f(a)\right|^{2}\left(1-|a|^{2}\right)^{2} 2 \int_{0}^{1}\left(1-r^{2}\right)^{p} r d r \\
& =\frac{1}{p+1} \sup _{a \in \mathbb{D}}\left|h^{\prime}(a) f(a)\right|^{2}\left(1-|a|^{2}\right)^{2} \geq \frac{1}{p+1}\left|h^{\prime}\left(z_{1}\right) f\left(z_{1}\right)\right|^{2}\left(1-\left|z_{1}\right|^{2}\right)^{2} \\
& \geq \frac{1}{p+1} \frac{\left|f\left(z_{1}\right)\right|^{2}}{\left\|g_{z_{1}}\right\|_{Q p}^{2}} .
\end{aligned}
$$

By Lemma 2.1 we have

$$
\left\|I_{f}\right\| \geq\left|f\left(z_{1}\right)\right|>c-\epsilon .
$$

Since $\epsilon$ is arbitrary, we have $\left\|I_{f}\right\| \geq \sup _{z \in \mathbb{D}}|f(z)|$ and the proof is complete.

Next, we consider the norm of $I_{f}$ from $Q_{p}(0<p<1)$ to $\mathfrak{B}$.

Theorem 2.3 Let $0<p<1$. If $f \in H(\mathbb{D})$, then $I_{f}$ is bounded from $Q_{p}$ space to $\mathfrak{B}$ space if and only iff $\in H^{\infty}$. Moreover, we have

$$
\left\|I_{f}\right\|=(p+1)^{1 / 2}\|f\|_{H^{\infty}}
$$

Proof If $f \in H^{\infty}$, then (1.2) gives

$$
\left\|I_{f} h\right\|_{\mathfrak{B}}=\sup _{z \in \mathbb{D}}\left|f(z) h^{\prime}(z)\right|\left(1-|z|^{2}\right) \leq\|f\|_{H^{\infty}} \sup _{z \in \mathbb{D}}\left|h^{\prime}(z)\right|\left(1-|z|^{2}\right) .
$$

From a part of the proof of estimate (2.2) for $f \equiv 1$, we see that

$$
\sup _{z \in \mathbb{D}}\left|h^{\prime}(z)\right|\left(1-|z|^{2}\right) \leq(p+1)^{1 / 2}\|h\|_{Q_{p}}
$$


and so

$$
\left\|I_{f} h\right\|_{\mathfrak{B}} \leq\|f\|_{H^{\infty}}(p+1)^{1 / 2}\|h\|_{Q_{p}} .
$$

This leads to

$$
\left\|I_{f}\right\| \leq(p+1)^{1 / 2}\|f\|_{H^{\infty}} .
$$

On the other hand, define $c=\sup _{z \in \mathbb{D}}|f(z)|$. Given any $\epsilon>0$, there exists $z_{1} \in \mathbb{D}$ such that $\left|f\left(z_{1}\right)\right|>c-\epsilon$. Let $h(z)=g_{z_{1}}(z) /\left\|g_{z_{1}}\right\|_{Q_{p}}$, where

$$
g_{z_{1}}(z)=\frac{z_{1}-z}{1-\bar{z}_{1} z}-z_{1}
$$

This together with Lemma 2.1 gives the following:

$$
\begin{aligned}
\left\|I_{f}\right\| & \geq\left\|I_{f} h\right\|_{\mathfrak{B}}=\sup _{z \in \mathbb{D}}\left|f(z) h^{\prime}(z)\right|\left(1-|z|^{2}\right) \geq\left|f\left(z_{1}\right) h^{\prime}\left(z_{1}\right)\right|\left(1-\left|z_{1}\right|^{2}\right) \\
& =\left|f\left(z_{1}\right)\right| /\left\|g_{z_{1}}\right\|_{Q_{p}}>(p+1)^{1 / 2}(c-\epsilon) .
\end{aligned}
$$

Since $\epsilon$ is arbitrary, we have

$$
\left\|I_{f}\right\| \geq(p+1)^{1 / 2} \sup _{z \in \mathbb{D}}|f(z)|=(p+1)^{1 / 2}\|f\|_{H^{\infty}} .
$$

The proof is complete.

Finally, we consider the norm of the integral operator $I_{f}$ on $\mathfrak{B}^{\alpha}, 0<\alpha<1$.

Theorem 2.4 Let $0<\alpha<1$ and $f \in H(\mathbb{D})$. Then the integral operator $I_{f}$ is bounded on $\mathfrak{B}^{\alpha}$ if and only if $f \in H^{\infty}$. Moreover,

$$
\left\|I_{f}\right\|=\|f\|_{H^{\infty}} .
$$

Proof For any $h \in \mathfrak{B}^{\alpha}$ with $\|h\|_{\mathfrak{B}^{\alpha}}=1$, by (1.3) we have

$$
\left\|I_{f} h\right\|_{\mathfrak{B}^{\alpha}}=\sup _{z \in \mathbb{D}}\left(1-|z|^{2}\right)^{\alpha}|f(z)|\left|h^{\prime}(z)\right| \leq\|h\|_{\mathfrak{B}^{\alpha}} \cdot\|f\|_{H^{\infty}} .
$$

This implies $\left\|I_{f}\right\| \leq\|f\|_{H^{\infty}}$.

Now we need to show the reverse inequality. Define $c=\sup _{z \in \mathbb{D}}|f(z)|$. Given any $\epsilon>0$, there exists $z_{1} \in \mathbb{D}$ such that $\left|f\left(z_{1}\right)\right|>c-\epsilon$. Put

$$
h(z)=\int_{\Gamma(z)} \frac{\left(1-\left|z_{1}\right|^{2}\right)^{\alpha}}{\left(1-\bar{z}_{1} \zeta\right)^{2 \alpha}} d \zeta,
$$

where $\Gamma(z)$ is any path in $\mathbb{D}$ from 0 to $z$, and a single-valued analytic branch is specified. By Theorem 13.11 in [36, p.274], we know $h$ is an analytic function in $\mathbb{D}$ and $h^{\prime}(z)=(1-$ 
$\left.\left|z_{1}\right|^{2}\right)^{\alpha} /\left(1-\bar{z}_{1} z\right)^{2 \alpha}$. Also, it is easy to check $\|h\|_{\mathfrak{B}^{\alpha}}=1$. In fact,

$$
\begin{aligned}
\|h\|_{\mathfrak{B}^{\alpha}} & =\sup _{z \in \mathbb{D}}\left|h^{\prime}(z)\right|\left(1-|z|^{2}\right)^{\alpha}=\sup _{z \in \mathbb{D}} \frac{\left(1-\left|z_{1}\right|^{2}\right)^{\alpha}}{\left|1-\bar{z}_{1} z\right|^{2 \alpha}}\left(1-|z|^{2}\right)^{\alpha} \\
& \leq \sup _{z \in \mathbb{D}} \frac{\left(1-\left|z_{1}\right|^{2}\right)^{\alpha}\left(1-|z|^{2}\right)^{\alpha}}{\left(1-\left|z_{1}\right||z|\right)^{2 \alpha}} \leq 1
\end{aligned}
$$

On the other hand, we have

$$
\begin{aligned}
\|h\|_{\mathfrak{B}^{\alpha}} & =\sup _{z \in \mathbb{D}}\left|h^{\prime}(z)\right|\left(1-|z|^{2}\right)^{\alpha}=\sup _{z \in \mathbb{D}} \frac{\left(1-\left|z_{1}\right|^{2}\right)^{\alpha}}{\left|1-\bar{z}_{1} z\right|^{2 \alpha}}\left(1-|z|^{2}\right)^{\alpha} \\
& \geq \frac{\left(1-\left|z_{1}\right|^{2}\right)^{\alpha}}{\left(1-\left|z_{1}\right|^{2}\right)^{2 \alpha}}\left(1-\left|z_{1}\right|^{2}\right)^{\alpha}=1 .
\end{aligned}
$$

Hence, the assertion follows by (2.4) and (2.5). Thus

$$
\begin{aligned}
\left\|I_{f}\right\| & \geq\left\|I_{f} h\right\|_{\mathfrak{B}^{\alpha}}=\sup _{z \in \mathbb{D}}\left|f(z) h^{\prime}(z)\right|\left(1-|z|^{2}\right)^{\alpha} \geq\left|f\left(z_{1}\right) h^{\prime}\left(z_{1}\right)\right|\left(1-\left|z_{1}\right|^{2}\right)^{\alpha} \\
& \geq\left|f\left(z_{1}\right)\right| \frac{\left(1-\left|z_{1}\right|^{2}\right)^{\alpha}}{\left(1-\left|z_{1}\right|^{2}\right)^{2 \alpha}}\left(1-\left|z_{1}\right|^{2}\right)^{\alpha}=\left|f\left(z_{1}\right)\right|>c-\epsilon .
\end{aligned}
$$

Since the $\epsilon$ is arbitrary, the proof is complete.

\section{Competing interests}

The authors declare that they have no competing interests.

\section{Authors' contributions}

All the authors contributed to the writing of the present article. They also read and approved the final manuscript.

\section{Author details}

'College of Mathematics and Information Science, Henan Normal University, Xinxiang, 453007, China. ${ }^{2}$ Department of Mathematics, JiaYing University, Meizhou, GuangDong 514015, China.

\section{Acknowledgements}

The first author is supported by the National Natural Science Foundation of China (No. 11126284). The second author is supported by the project of Department of Education of Guangdong Province (No. 2012KJCX0096).

\section{Received: 21 April 2013 Accepted: 10 July 2013 Published: 25 July 2013}

\section{References}

1. Aulaskari, R, Xiao, J, Zhao, R: On subspaces and subsets of BMOA and UBC. Analysis 15, 101-121 (1995)

2. Xiao, J: Holomorphic $\mathcal{Q}$ Classes. Lecture Notes in Math., vol. 1767. Springer, Berlin (2001)

3. Zhu, K: Operator Theory in Function Spaces. Dekker, New York (1990)

4. Zhu, K: Bloch type spaces of analytic functions. Rocky Mt. J. Math. 23, 1143-1177 (1993)

5. Aleman, A, Siskakis, A: An integral operator on $H^{p}$. Complex Var. Theory Appl. 28, 140-158 (1995)

6. Aleman, A, Siskakis, A: Integral operators on Bergman spaces. Indiana Univ. Math. J. 46, 337-356 (1997)

7. Austin, A: Multiplication and integral operators on Banach spaces of analytic functions. Ph.D. thesis, University of Hawai (2010)

8. Krantz, S, Stević, S: On the iterated logarithmic Bloch space on the unit ball. Nonlinear Anal. TMA 71, 1772-1795 (2009)

9. Li, S, Stević, S: Volterra-type operators on Zygmund spaces. J. Inequal. Appl. 2007, Article ID 32124 (2007)

10. Li, S, Stević, S: Integral type operators from mixed-norm spaces to $\alpha$-Bloch spaces. Integral Transforms Spec. Funct. 18(7), 485-493 (2007)

11. Li, S, Stević, S: Riemann-Stieltjes operators between different weighted Bergman spaces. Bull. Belg. Math. Soc. Simon Stevin 15(4), 677-686 (2008)

12. Li, S, Stević, S: Products of composition and integral type operators from $H^{\infty}$ to the Bloch space. Complex Var. Elliptic Equ. 53(5), 463-474 (2008)

13. Li, S, Stević, S: Generalized composition operators on Zygmund spaces and Bloch type spaces. J. Math. Anal. Appl. $338,1282-1295$ (2008) 
14. Li, S, Stević, S: Products of integral-type operators and composition operators between Bloch-type spaces. J. Math. Anal. Appl. 349, 596-610 (2009)

15. Li, S: On an integral-type operator from the Bloch space into the $Q_{K}(p, q)$ space. Filomat $26,125-133(2012)$

16. Pan, C: On an integral-type operator from $Q_{K}(p, q)$ spaces to $\alpha$-Bloch space. Filomat $25,163-173(2011)$

17. Pommerenke, C: Schlichte funktionen und analytische funktionen von beschränkter mittlerer oszillation. Comment. Math. Helv. 52, 591-602 (1977)

18. Stević, S: On a new operator from the logarithmic Bloch space to the Bloch-type space on the unit ball. Appl. Math. Comput. 206, 313-320 (2008)

19. Stević, S: On a new integral-type operator from the weighted Bergman space to the Bloch-type space on the unit ball. Discrete Dyn. Nat. Soc. 2008, Article ID 154263 (2008)

20. Stević, S: On a new integral-type operator from the Bloch space to Bloch-type spaces on the unit ball. J. Math. Anal. Appl. 354, 426-434 (2009)

21. Zhu, X: An integral-type operator from $H^{\infty}$ to Zygmund-type spaces. Bull. Malays. Math. Soc. 35, $679-686$ (2012)

22. Bourdon, P, Fry, E, Hammond, C, Spofford, C: Norms of linear-fractional composition operators. Trans. Am. Math. Soc. 356, 2459-2480 (2003)

23. Colonna, F, Easley, G, Singman, D: Norm of the multiplication operators from $H^{\infty}$ to the Bloch space of a bounded symmetric domain. J. Math. Anal. Appl. 382,621-630 (2011)

24. Hammond, C: The norm of a composition operator with linear symbol acting on the Dirichlet space. J. Math. Anal. Appl. 303, 499-508 (2005)

25. Liu, J, Lou, Z, Xiong, C: Essential norms of integral operators on spaces of analytic functions. Nonlinear Anal. 75, 5145-5156 (2012)

26. Martín, M: Norm-attaining composition operators on the Bloch spaces. J. Math. Anal. Appl. 369, 15-21 (2010)

27. Stević, S: Norm of weighted composition operators from Bloch space to $H_{\mu}^{\infty}$ on the unit ball. Ars Comb. 88, 125-127 (2008)

28. Stević, S: Norms of some operators from Bergman spaces to weighted and Bloch-type spaces. Util. Math. 76, 59-64 (2008)

29. Stević, S: Norm of weighted composition operators from $\alpha$-Bloch spaces to weighted-type spaces. Appl. Math. Comput. 215, 818-820 (2009)

30. Stević, S: Norm and essential norm of composition followed by differentiation from $\alpha$-Bloch spaces to $H_{\mu}^{\infty}$. Appl. Math. Comput. 207, 225-229 (2009)

31. Stević, S: Norms of some operators on bounded symmetric domains. Appl. Math. Comput. 215, 187-191 (2010)

32. Stević, S: Norm of an integral-type operator from Dirichlet to Bloch space on the unit disk. Util. Math. 83, 301-303 (2010)

33. Stević, S: On an integral operator between Bloch-type spaces on the unit ball. Bull. Sci. Math. 134, $329-339$ (2010)

34. Yang, W: On an integral-type operator between Bloch-type spaces. Appl. Math. Comput. 215, 954-960 (2009)

35. Liu, J, Xiong, C: Norm-attaining integral operators on analytic function spaces. J. Math. Anal. Appl. 399, 108-115 (2013)

36. Rudin, W, Real and Complex Analysis, 3rd edn. McGraw-Hill, New York (1987)

doi:10.1186/1029-242X-2013-342

Cite this article as: Li and Li: Norm of an integral operator on some analytic function spaces on the unit disk. Journal of Inequalities and Applications 2013 2013:342.

\section{Submit your manuscript to a SpringerOpen ${ }^{\circ}$ journal and benefit from:}

- Convenient online submission

Rigorous peer review

- Immediate publication on acceptance

Open access: articles freely available online

- High visibility within the field

- Retaining the copyright to your article 